\title{
Management strategies for acute STEMI in low- and middle- income countries: experience of the Tamil Nadu ST-segment elevation myocardial infarction programme
}

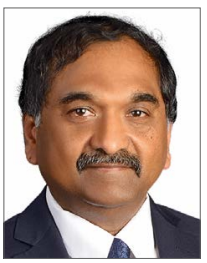

Thomas Alexander ${ }^{*}$, MD, DM; Ajit S. Mullasari², MD, DM;

Brahmajee K. Nallamothu, MD, MPH

1. Kovai Medical Centre and Hospital, Coimbatore, India; 2. Department of Cardiology, Madras Medical Mission, Chennai, India; 3. Department of Internal Medicine, University of Michigan, Ann Arbor, MI, USA

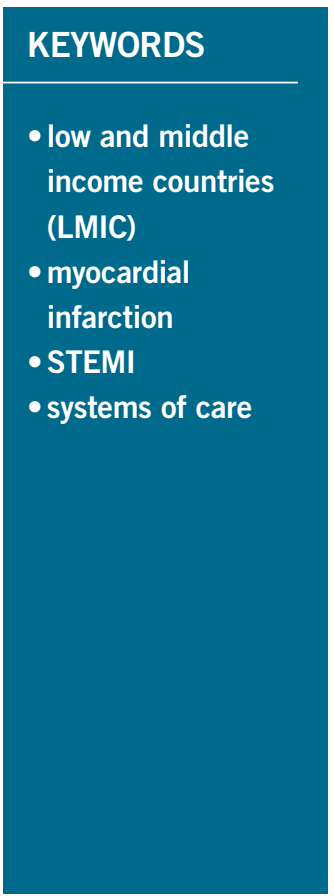

\section{Abstract}

Appropriate and timely management of ST-elevation myocardial infarction is a major challenge in developing countries due to inadequate infrastructure and trained manpower. The TN-STEMI Program was a successful STEMI system of care that was run in the South Indian state of Tamil Nadu. Lessons learnt from this programme could help to understand the challenges and provide solutions to running similar programmes in low- and middle-income countries.

*Corresponding author: Kovai Medical Centre and Hospital, 99 Avanashi Rd, Coimbatore, Tamil Nadu, 641014, India. 


\section{Abbreviations}

BPL

LMIC

NCD

PCI

STEMI

TN-STEMI Program

below poverty level

low- and middle-income countries

non-communicable disease

percutaneous coronary intervention

ST-elevation myocardial infarction

dial infarction programme

\section{Introduction}

ST-elevation myocardial infarction (STEMI) is a subset of acute coronary syndromes that requires immediate and optimal reperfusion therapy to reduce morbidity and mortality and optimise myocardial salvage. Data show that reperfusion achieved within 12 hours of onset of symptoms and preferably within the first hour results in maximal benefit ${ }^{1}$. Management protocols, STEMI networks and universal access to reperfusion therapy in developed countries have ensured that the mortality and morbidity have been significantly reduced. Low- and middle-income countries (LMIC), unfortunately, do not have the resources, manpower or infrastructure to emulate the ideal management of STEMI. Added to this is the increased burden of STEMI in many of these countries ${ }^{2}$ and the younger age of presentation ${ }^{3,4}$. Gender and economic disparity in access and healthcare delivery also results in increased morbidity and mortality ${ }^{5,6}$, emphasising the need for designing systems to ensure equity in delivery of the appropriate reperfusion therapy.

The Tamil Nadu ST-segment elevation myocardial infarction programme (TN-STEMI Program) has been a successful STEMI programme run in the South Indian state of Tamil Nadu. The model and the critical components of the STEMI network could help to understand and plan similar programmes in countries with fewer resources.

Editorial, see page 11

\section{THE STEMI INDIA MODEL}

Studies from LMIC have shown variable rates of reperfusion therapy in STEMI patients. Certain urban registries have shown reperfusion rates of $60 \%{ }^{7,8}$; however, rural data from India show reperfusion rates as low as $36.2 \%$. Most of these patients receive stand-alone thrombolysis. Many of the LMIC would probably show similar results ${ }^{10}$.

Primary percutaneous coronary intervention (PCI), when delivered in a timely fashion, is the preferred mode of reperfusion, recommended by all international guidelines ${ }^{1,11}$. Despite the fact that LMIC recognise the need to increase primary PCI facilities and that there has been a steady increase in the number of primary PCIs carried out in LMIC, only a small minority of STEMI patients receive this modality of reperfusion ${ }^{7}$. Thrombolysis is much more widely available and can be safely administered under medical supervision. Thrombolysis can then be followed by systematic angiography within 2-24 hours, and PCI if appropriate. This approach that combines thrombolysis with PCI is called pharmaco-invasive management. It has been shown to be superior to stand-alone thrombolysis and ischaemia-directed angiography in multiple studies ${ }^{12}$. This has been endorsed by multiple guidelines including those of the European Society of Cardiology. The STREAM (STrategic Reperfusion Early After Myocardial infarction) data $^{13}$ and the Indian data from the STEPP-AMI study ${ }^{14}$ showed that the pharmaco-invasive strategy compared well with primary PCI in terms of overall morbidity and mortality.

Based on this evidence, STEMI India proposed that STEMI management in India adopt the dual strategy of combining primary PCI with pharmaco-invasive reperfusion (Figure 1) to develop an easily implementable hub-and-spoke STEMI system of care, referred to as the STEMI India model $^{15}$ (Figure 2). It is based on two fundamental principles:

1. Primary PCI for STEMI patients located close to designated hub hospitals with catheterisation laboratories. This would cater

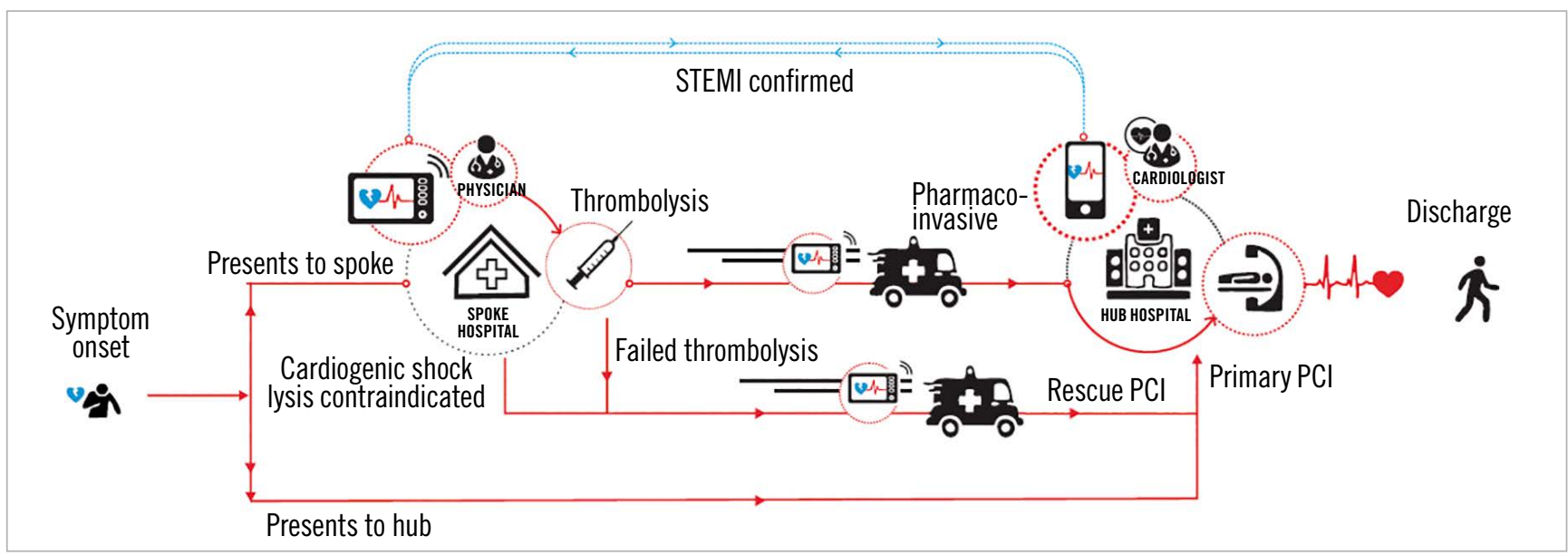

Figure 1. The two treatment pathways that a STEMI patient may take depending on whether the initial presentation is at the "hub" hospital or "spoke" hospital. 


\section{The STEMI India model}
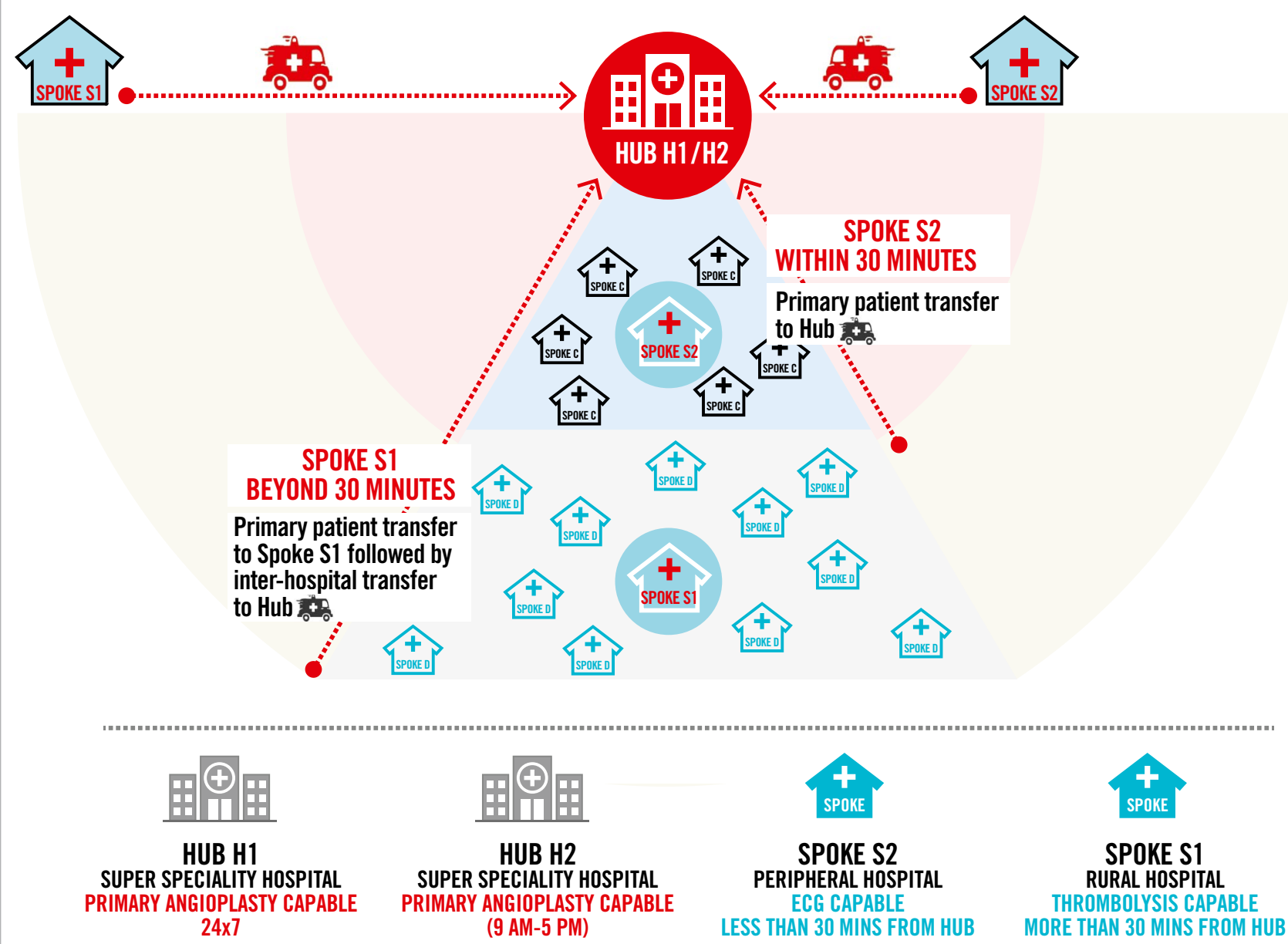

Figure 2. The "hub-and-spoke" model. H1 hub is a $24 / 7$ primary PCI-capable hospital while an H2 hub has primary PCI capability but is not available 24/7. S1 spoke is usually located beyond 30 min driving distance from a hub hospital and is thrombolysis capable. An $S 2$ spoke is usually located within 30 minutes driving distance to a hub and transfers a patient directly to the hub for primary PCI after ECG confirmation of STEMI. Primary ambulance pick-up and transfer will be to a hub hospital (H1 or H2) or to an S1 spoke but not to an S2 spoke.

mostly to patients in urban areas with short transportation time (less than 30 minutes).

2. Patients in rural areas and located away from the hub hospitals (more than 30 minutes to the hub) would be moved to thrombolysis-capable spoke hospitals for initial thrombolysis and then moved within 2-24 hours to the hub hospital for catheterisation and PCI, if indicated (pharmaco-invasive treatment).

\section{TN-STEMI PROGRAM}

The details of the TN-STEMI Program have been reported earlier ${ }^{16}$. The study had the active participation of the local government, the state ambulance network (appointed by the government) and the government-funded health insurance scheme for "below poverty level" (BPL) citizens.

The programme aimed to create an integrated, regional quality improvement programme that linked 35 thrombolysis-capable spoke healthcare centres to four large PCI-capable hub hospitals (each hub - and the connected spokes - was called a cluster), thus demarcating four clusters.

The reperfusion strategy was focused on increasing the two proven superior reperfusion strategies of primary PCI in the hub hospitals and pharmaco-invasive treatment in the spoke hospitals, and at the same time reducing the number of no reperfusion and stand-alone fibrinolysis.

Primary outcomes focused on the proportion of patients undergoing reperfusion, timely reperfusion, and post-fibrinolysis angiography and PCI. Secondary outcomes were in-hospital and one-year mortality.

A total of 2,420 patients with STEMI (2,034 men [84.0\%] and 386 women [16.0\%]; mean [SD] age, 54.7 [12.2] years) (898 in the pre-implementation phase and 1,522 in the post-implementation phase) were enrolled. 
Overall reperfusion use and times to reperfusion were similar (795 [88.5\%] vs 1,372 [90.1\%]; $\mathrm{p}=0.21$ ). Coronary angiography (314 [35.0\%] vs 925 [60.8\%]; p<0.001) and PCI (265 [29.5\%] vs $707[46.5 \%] ; p<0.001)$ were more commonly performed during the post-implementation phase. In-hospital mortality was not different (52 [5.8\%] vs 85 [5.6\%]; $\mathrm{p}=0.83)$, but one-year mortality was lower in the post-implementation phase (134 [17.6\%] vs 179 [14.2\%]; $\mathrm{p}=0.04)$. This difference remained consistent after multivariable adjustment (adjusted odds ratio [OR] 0.76, 95\% CI: 0.58-0.98; $\mathrm{p}=0.04$ ).

Taken together, the in-hospital mortality rate for STEMI patients receiving no reperfusion therapy was $10.18 \%$ and for those with stand-alone thrombolysis was $9.04 \%$. Using propensity matching and multivariable models, the 400 patients who had pharmacoinvasive treatment with streptokinase were compared with the 815 patients who received primary $\mathrm{PCI}^{17}$. The in-hospital mortality was much lower and similar for these two reperfusion methods (2.0 vs $2.1 \%$; OR 2.05, 95\% CI: 0.47-8.93; p=0.34). Furthermore, the one- and two-year mortality rates were also similar with similar in-hospital bleeding.

The rates of stand-alone thrombolysis came down significantly in the programme. The use of primary PCI or pharmaco-invasive reperfusion treatment increased during the post-implementation period (191 of 413 [46.3\%] vs 601 of 954 [63\%]; $p=0.001$ ). This was especially significant in the spokes which showed an increase in pharmaco-invasive treatment (17 of 485 [3.5\%] vs 178 of 568 [31.3\%]; $p=0.001$ ).

Given that there was no change in reperfusion times, the reduction in mortality can be attributed entirely to the greater utilisation of the two superior reperfusion treatments of primary PCI and the pharmaco-invasive treatment.

Subgroup analysis of gender differences in the STEMI patients in the TN-STEMI Program showed that symptom to first medical contact times in female patients were significantly longer compared to male patients (193 minutes vs 170 minutes, $\mathrm{p} \leq 0.009)$. Women had higher mortality, both in-hospital (10.4\% vs $4.8 \%$; $\mathrm{p} \leq 0.001)$ and at one year $(26.7 \%$ vs $13 \% ; \mathrm{p} \leq 0.001)$. As was seen in the whole group, there was no significant reduction in ischaemia times in women between the pre- and post-implementation subsets. However, the increased use of the superior reperfusion treatments of primary PCI and pharmaco-invasive treatment resulted in a significant reduction in one-year mortality $(33 \%$ vs $22.6 \%$; $\mathrm{p}=0.03$ ), though still considerably higher than the mortality for men.

A cost-benefit analysis ${ }^{18}$ of the programme was carried out based on the investment for the programme and the lives saved within the geography of the study. Considering the benefits accrued only by mortality reduction, the cost-benefit ratio is 3.98 . If the benefits accrued due to lower morbidity are added, the cost benefit ratio would strengthen. This showed that the STEMI India model is a cost-effective intervention. This should be of considerable interest to policymakers in LMIC where financial and resource constraints are a huge public health challenge.
The project was successful on several levels:

- At the individual level: as users of the healthcare system, patients were beneficially impacted by the intervention, with outcomes of mortality and morbidity of patients improving.

- At the institutional level: at the infrastructure level, more patients accessed PCI through the pharmaco-invasive treatment during the intervention as compared to the base case without any increase in fixed costs, indicating that the infrastructure throughput was increased. Furthermore, from the perspective of human resource infrastructure, there was considerable capacity building for staff at spokes, which helped to train these staff to understand and deal with STEMI patients in a better and more appropriate fashion.

- At the society level: as demonstrated in the cost-benefit analysis, the programme was economically beneficial for the state and successful in improving the lives of the citizens that it impacted.

\section{UNIQUE FEATURES FOR THE SUCCESS OF THE PROGRAMME}

The success of the programme can be attributed to five factors which are extremely important:

\section{A DEDICATED AGENCY FOR PROGRAMME IMPLEMENTATION}

STEMI India was a dedicated organisation running the programme: a dedicated team ensured collaboration among the hospitals, the ambulance network and the insurance agencies. Protocols were defined and monitored for adherence, the necessary periodic training was organised, patient data collection and analysis was provided, post-treatment patient follow-up was undertaken, and issues and gaps were identified and addressed.

\section{COLLABORATION WITH THE STATE GOVERNMENT}

Health is a state subject in India and, even though private healthcare infrastructure accounts for $65 \%$ of total healthcare infrastructure in India, the vast majority of patients, especially those from the lower socio-economic strata, access public hospitals for health care. These public hospitals are under the control of the government health department and buy-in from the government ensured participation of these hospitals and benefited this group of patients. The collaboration with the government also helped in two other important factors listed below.

\section{PARTNERSHIP WITH AMBULANCE INFRASTRUCTURE}

The 108 ambulance service is funded and controlled by each state government but is run by private entities under a public-private collaboration. Through the collaboration with the local government, STEMI India could train and utilise the ambulance services for primary pick-up and inter-hospital transfers of STEMI patients. SOCIAL HEALTH INSURANCE

One of the unique insurance programmes run by the state government is the insurance for the BPL population. Under this programme, the targeted population is given health insurance cover for several diseases including STEMI treatment. The insurance premium is paid for by the government. By linking this to the STEMI programme, it was possible to cover $49 \%$ of the patients in the post-implementation period. Many of these 
patients would not have been able to access this care without the BPL insurance.

\section{ECG/DATA ENTRY AND TRANSMISSION INFRASTRUCTURE}

It was critical to combine real-time data entry with the ability to transmit an electrocardiogram (ECG) to the hub hospital for STEMI confirmation. This, indigenously developed low-cost device, ensured quick confirmation of STEMI so that the less experienced spoke hospital physician could initiate thrombolysis. This also captured data for audit, analysis, and quality improvement purposes.

\section{UNADDRESSED ISSUES}

There are a few inadequately addressed issues that could have improved the results of this programme.

- Pre-hospital delay. The median delay in the study was 170 minutes versus 174 minutes in the post-implementation period. This did not change significantly. This is critically dependent on patient behaviour and ambulance availability. Multiple strategies have been implemented in various countries with mixed results ${ }^{19}$. A dedicated public education programme was not part of the project - this could have helped.

- The "no reperfusion" group remained largely the same with only a modest decrease with initiation of the programme $(11.5 \%$ vs $9.9 \% ; p=0.21$ ). These are typically patients who present too late for reperfusion or die prior to the initiation of reperfusion therapy. A small proportion did not come under the health insurance umbrella and so could not access care. A sustained campaign for early recognition of STEMI symptoms could reduce these numbers.

- The gender disparity was not specifically addressed. The delayed presentation of women and the increased mortality as compared to men require special attention and targeted interventions. Though there was improvement in the post-implementation one-year mortality rates, this was still very much higher than that for their male counterparts.

- Primary ambulance pick-up rates were very low, especially in the rurally located spoke hospitals. The 108 ambulance services have hitherto prioritised pick-up and transfer of accident victims and pregnancy and child health cases. A sustained campaign to educate the public about the availability and critical importance of ambulance transfer is required.

- The time from ECG to fibrinolysis (median in minutes) between pre and post implementation was 30 (10-80) versus 25 (15-45); $\mathrm{p}=0.19$, and door to balloon in the primary PCI group of 100 (84-143) versus 105 (80-145). These are reasonable timings; further reduction is difficult considering the medical facilities' ability to handle these patients as well as the delay in insurance approvals or committing the finances by patients who pay out of pocket.

- The increase in the superior pharmaco-invasive reperfusion method in the spokes, though showing almost a tenfold increase $(3.5 \%$ vs $31.3 \%$; $<<0.001)$ over the mean nine-month implementation period, is still very low. This could increase over a longer period of implementing such a programme. Issues also include the reluctance of rural patients to be transferred to a city-based hub hospital for invasive procedures.

\section{A UNIFIED STRATEGY FOR SUCCESS}

From our experience in running this programme and subsequently a few other programmes across India, we have captured what we believe is needed, which is a unified strategy for success with the collaborative participation of the public and private sectors.

\section{STATE GOVERNMENT BUY-IN}

The state government's will to implement a statewide STEMI programme is probably the most critical and difficult component in initiating a STEMI programme in LMIC. Since governments control many of the key components of a programme and public hospital participation is critical to equity in STEMI care delivery, it is imperative that there is a buy-in from governments. Bureaucratic lethargy and long-serving government officials who are resistant to any innovation that comes from outside the system make it difficult to change the status quo. Educating the political and bureaucratic machinery and the time and effort required for this can be very frustrating, especially when, as is often the case in India, a change in personnel or political leadership requires a restart of the whole process. PUBLIC EDUCATION

Successful strategies need to be tailored to the local social and cultural milieu and should consider the educational status of the target audience. Targeted strategies crafted to address the gender gap should also be a priority. Strategies that could work include:

- Multimedia campaigns through local TV channels and FM radio utilising local language and themes.

- Targeting the vulnerable population, for example, patients attending non-communicable disease (NCD) clinics.

- Increasing awareness among school children, especially those whose parents are from a lower socio-economic or educational background, may help to spread the message to this group of patients.

These messages should be part of a sustained campaign rather than for short periods. Focusing on typical symptoms in the elderly and women will also improve early presentation of this subgroup.

\section{FINANCING REPERFUSION THERAPY}

This is a major barrier to timely treatment in LMIC. The challenge to fund treatment for the large numbers of BPL patients in LMIC can be tackled utilising some form of social insurance scheme, such as the PMJAY scheme ${ }^{20}$ in India. While lytic therapy may be available free of cost in public hospitals, the cost of the complete treatment including ambulance transfer and PCI, when appropriate, should be included in BPL insurance schemes. These schemes should also ensure financing the post-discharge medications and consider integrating this with app-based follow-up to ensure medication compliance and lifestyle advice.

\section{SELECTION OF AN APPROPRIATE MODEL OF CARE}

The STEMI India model of combining primary PCI with pharmaco-invasive treatment in a hub-and-spoke fashion may be 
appropriate in urban and semi-urban locations. However, more remote areas and those without basic infrastructure may need to be developed initially as purely thrombolytic centres. These could require support from tele-ECG services or even a tele-CCU service. However, with improvement in infrastructure and manpower, some of these can migrate into a fully functional hub-andspoke model.

\section{AMBULANCE INFRASTRUCTURE}

Enabling a unified protocol across the state-nominated ambulance network will ensure that STEMI patients receive appropriate care in the ambulances, both during primary pick-up and transfer to a STEMI-ready hospital and during inter-facility transfer.

\section{SPOKE INFRASTRUCTURE}

- Legislative and regulatory measures, in association with local scientific bodies, could accredit hospitals to manage STEMI based on infrastructure, manpower and training criteria. Mandatory process-of-care measures for quality improvement and public reporting of outcomes could result in development of a robust STEMI programme.

- STEMI-ready hospitals: designation of participating hospitals as "Heart Attack Accredited Centres" helps to ensure that patients in that geography know where to go in case of chest pain. This would reduce delay and ensure protocol-based treatment and prompt reperfusion and appropriate triage.

\section{INTEGRATION OF HOSPITALS IN PATIENT CARE}

Many of the LMIC have both public and private hospitals taking care of healthcare needs. The availability of tertiary care and cardiac catheterisation laboratories in most LMIC is significantly greater in private facilities as compared to public hospitals. However, the public hospitals cater to most BPL patients, resulting in overcrowding and inability to prioritise STEMI treatment. Utilisation of spare capacity in the private sector, linking it to some form of social insurance and making it part of a STEMI network in a public-private partnership, could help to ramp up the programme rapidly.

\section{AN INDEPENDENT STEMI COORDINATING AGENCY/TASK FORCE}

An independent agency, team or department with capability to work with the various government entities and departments, ambulance services and the different types of hospital was and is critical to run a well-coordinated programme. This is because this team needs to look critically at patient outcomes at each health facility and avoid the risk of "going native". Data collection, gap analysis and monthly report generation as well as regular training programmes are required to ensure a smooth operation of the programme and ensure the continuous improvement of each participating entity. A single implementing agency has the added benefit of ensuring that, with improvement in infrastructure, the migration from purely thrombolytic centres to a hub-and-spoke model can be seamlessly implemented.

\section{STEMI DIAGNOSIS}

Diagnosis of STEMI, in the ambulance by a paramedic or in a spoke hospital by a primary care doctor who only occasionally manages ACS patients, is difficult. There is always some reluctance to transfer to an appropriate medical facility or to initiate lytic therapy. Transmission of the ECG to the hub hospital for STEMI confirmation - as done in the TN-STEMI Program - or utilising a dedicated tele-ECG service could be useful and reduce delays. There are multiple commercially available ECG machines with built-in transmission capability and STEMI India utilises a secure application for mobile communication.

\section{TRAINING PROGRAMMES FOR CAPACITY BUILDING}

Training and capacity building is critical for the success of the STEMI programme. The training requirements for spokes and hubs are entirely different. Spokes require more intensive and frequent training, while hub hospitals, which are already skilled at thrombolysis and PCI, require only a yearly update of newer techniques and guidelines. Undoubtedly, the importance of this component is the greatest in spokes which are currently not thrombolysing but require training of both doctors and nurses to develop into a thrombolysing spoke. Since staff transfers and attrition rates are high, periodic refresher courses are often needed.

\section{DATA AUDIT AND ANALYSIS}

A digital data entry software with real-time analytics was developed by STEMI India and used in the TN-STEMI Program and was critical for quality audit and gap analysis. Monthly reports to the spokes and hubs were generated using this and this helped the programme to run smoothly. This software is currently being used in programmes in other states in the country and outside.

\section{COORDINATION AND STAKEHOLDER ENGAGEMENT}

Periodic meeting of the various stakeholders is critical to the success of the programme. Periodic visits by the "hub" doctors to the spokes help to improve coordination as does prompt feedback after each transfer of a patient to the hub. A monthly stakeholder meeting needs to be held, even if it is web-based, to discuss issues, present results and solve problems.

The success of a programme of this magnitude where multiple activities are to be carried out simultaneously requires an umbrella implementation committee comprising the important stakeholders. This is critical to ensure smooth operations and to ensure rapid resolutions of problems. Commitment and participation of the governments, both at the centre and locally, are critical to implementation and to ensuring equity in delivery of STEMI care.

\section{Conclusions}

The TN-STEMI Program combined the two superior reperfusion modalities of pharmaco-invasive treatment with primary PCI in a hub-and-spoke model. This programme demonstrated a significant reduction in one-year mortality within nine months of starting the programme. The early reduction in mortality - the low hanging fruit - can come from change in management strategy from stand-alone thrombolysis to pharmaco-invasive and primary PCI treatment. Improving delays in accessing medical care and reducing system delays, which impacts on morbidity and mortality, will be a much more difficult and longer process 
that requires multiple interventions including public education, training of medical teams and use of digital tools, apart from improving ambulance services. A single implementing agency could be critical in coordinating the components of a successful STEMI system of care.

\section{Conflict of interest statement}

B.K. Nallamothu is a principal investigator or co-investigator on research grants from the NIH, VA HSR\&D, the American Heart Association, and Janssen. He also receives compensation as Editor-in-Chief of Circulation: Cardiovascular Quality \& Outcomes, a journal of the American Heart Association. Finally, he is a co-inventor on U.S. Utility Patent Number US15/356,012 (US20170148158A1) entitled "Automated Analysis of Vasculature in Coronary Angiograms" that uses software technology with signal processing and machine learning to automate the reading of coronary angiograms, held by the University of Michigan. The patent is licensed to AngioInsight, Inc., in which B.K. Nallamothu holds ownership shares and receives consultancy fees. The other authors have no conflicts of interest to declare.

\section{References}

1. Ibanez B, James S, Agewall S, Antunes MJ, Bucciarelli-Ducci C, Bueno H, Caforio ALP, Crea F, Goudevenos JA, Halvorsen S, Hindricks G, Kastrati A, Lenzen MJ, Prescott E, Roffi M, Valgimigli M, Varenhorst C, Vranckx P, Widimský P; ESC Scientific Document Group. 2017 ESC Guidelines for the management of acute myocardial infarction in patients presenting with ST-segment elevation: The Task Force for the management of acute myocardial infarction in patients presenting with ST-segment elevation of the European Society of Cardiology (ESC). Eur Heart J. 2018;39: 119-77.

2. Joseph P, Leong D, McKee M, Anand SS, Schwalm JD, Teo K, Mente A, Yusuf S. Reducing the Global Burden of Cardiovascular Disease, Part 1: The Epidemiology and Risk Factors. Circ Res. 2017;121:677-94.

3. Vedanthan R, Seligman B, Fuster V. Global perspective on acute coronary syndrome: a burden on the young and poor. Circ Res. 2014;114:1959-75.

4. Alexander T, Kumbhani DJ, Subban V, Sundar H, Nallamothu BK, Mullasari AS. Acute ST-Elevation Myocardial Infarction in the Young Compared With Older Patients in the Tamil Nadu STEMI Program. Heart Lung Circ. 2021 June 1. [Epub ahead of print].

5. Khraishah H, Alahmad B, Alfaddagh A, Jeong SY, Mathenge N, Kassab MB, Kolte D, Michos ED, Albaghdadi M. Sex disparities in the presentation, management and outcomes of patients with acute coronary syndrome: insights from the ACS QUIK trial. Open Heart. 2021;8:e01470.

6. Alexander T, Victor SM, Jayakumaran B, Rajan S, Mullasari AS. Sex-related Differences in Outcomes for Patients With ST-Elevation Myocardial Infarction (STEMI): A TN-STEMI Program Subgroup Analysis. Heart Lung Circ. 2021 June 2. [Epub ahead of print].
7. Xavier D, Pais P, Devereaux P, Xie C, Prabhakaran D, Reddy KS, Gupta R, Joshi P, Kerkar P, Thanikachalam S, Haridas K, Jaison T, Naik S, Maity A, Yusuf S; CREATE registry investigators. Treatment and outcomes of acute coronary syndromes in India (CREATE): a prospective analysis of registry data. Lancet. 2008; 371:1435-42.

8. Mohanan PP, Mathew R, Harikrishnan S, Krishnan MN, Zachariah G, Joseph J, Eapen K, Abraham M, Menon J, Thomas M, Jacob S, Huffman MD, Prabhakaran D; Kerala ACS Registry Investigators. Presentation, management, and outcomes of 25748 acute coronary syndrome admissions in Kerala, India: results from the Kerala ACS Registry. Eur Heart J. 2013;34:121-9.

9. Chand Negi P, Mahajan K, Merwaha R, Asotra S, Sharma R. Epidemiological trends of acute coronary syndrome in Shimla district of the hilly state of Northern India: Six-year data from the prospective Himachal Pradesh acute coronary syndrome registry. Indian Heart J. 2019;71:440-5.

10. Nascimento BR, Brant LCC, Marino BCA, Passaglia LG, Ribeiro ALP. Implementing myocardial infarction systems of care in low/middle-income countries. Heart. 2019;105:20-6.

11. O'Gara PT, Kushner FG, Ascheim DD, Casey DE Jr, Chung MK, de Lemos JA, Ettinger SM, Fang JC, Fesmire FM, Franklin BA, Granger CB, Krumholz HM, Linderbaum JA, Morrow DA, Newby LK, Ornato JP, Ou N, Radford MJ, Tamis-Holland JE, Tommaso JE, Tracy CM, Woo YJ, Zhao DX; CF/AHA Task Force. 2013 ACCF/AHA guideline for the management of ST-elevation myocardial infarction: executive summary: a report of the American College of Cardiology Foundation/American Heart Association Task Force on Practice Guidelines. Circulation. 2013;127:529-55. 12. Fazel R, Joseph TI, Sankardas MA, Pinto DS, Yeh RW, Kumbhani DJ, Nallamothu BK. Comparison of Reperfusion Strategies for ST-Segment-Elevation Myocardial Infarction: A Multivariate Network Meta-analysis. J Am Heart Assoc. 2020;9:e 015186.

13. Armstrong PW, Gershlick AH, Goldstein P, Wilcox R, Danays T, Lambert Y, Sulimov V, Rosell Ortiz F, Ostojic M, Welsh RC, Carvalho AC, Nanas J, Arntz HR, Halvorsen S, Huber K, Grajek S, Fresco C, Bluhmki E, Regelin A, Vandenberghe K, Bogaerts K, Van de Werf F. Fibrinolysis or primary PCI in ST-segment elevation myocardial infarction. N Engl J Med. 2013;368:1379-87.

14. Victor SM, Subban V, Alexander T, Bahuleyan CG, Srinivas A, Selvamani S, Mullasari AS. A prospective, observational, multicentre study comparing tenecteplase facilitated PCI versus primary PCI in Indian patients with STEMI (STEPP-AMI). Open Heart. 2014;1:e000133.

15. Alexander T, Mullasari AS, Kaifoszova Z, Khot UN, Nallamothu B, Ramana RGV, Sharma M, Subramaniam K, Veerasekar G, Victor SM, Chand K, Deb PK, Venugopal K, Chopra HK, Guha S, Banerjee AK, Armugam AM, Panja M, Wander GS. Framework for a National STEMI Program: Consensus document developed by STEMI INDIA, Cardiological Society of India and Association Physicians of India. Indian Heart J. 2015;67:497-502.

16. Alexander T, Mullasari AS, Joseph G, Kannan K, Veerasekar G, Victor SM, Ayers C, Thomson VS, Subban V, Gnanaraj JP, Narula J, 
Kumbhani DJ, Nallamothu BK. A System of Care for Patients With ST-Segment Elevation Myocardial Infarction in India: The Tamil Nadu-ST-Segment Elevation Myocardial Infarction Program. JAMA Cardiol. 2017;2:498-505.

17. Kumbhani DJ, Alexander T, Nallamothu BK, Menon V, Ayers C, Mullasari AS; TN-STEMI Investigators. Pharmacoinvasive Approach with Streptokinase in Low to Intermediate Risk ST-Elevation Myocardial Infarction Patients: Insights from the Tamil Nadu-STEMI Initiative. Am J Cardiovasc Drugs. 2019;19: 517-9.

18. Mohan VN, Alexander T, Muraleedharan VR, Mullasari A, Narula J, Khot UN, Nallamothu BK, Kumbhani DJ. Economic and Societal Impact of a Systems-of-Care Approach for STEMI
Management in Low and Middle-Income Countries: Insights from the TN STEMI Program. Ann Glob Health. 2019;85:122.

19. Pereira H, Calé R, Pinto FJ, Pereira E, Caldeira D, Mello S, Vitorino S, Almeida MS, Mimoso J; centers participating in the Stent for Life Initiative Portugal. Factors influencing patient delay before primary percutaneous coronary intervention in ST-segment elevation myocardial infarction: The Stent for life initiative in Portugal. Rev Port Cardiol. 2018;37:409-21.

20. Garg S, Bebarta KK, Tripathi N. Performance of India's national publicly funded health insurance scheme, Pradhan Mantri Jan Arogaya Yojana (PMJAY), in improving access and financial protection for hospital care: findings from household surveys in Chhattisgarh state. BMC Public Health. 2020;20:949. 